\title{
A New Era
}

want to remind you that this will be the last printed

issue of Queue-but also to reassure you that Queue is

not going away. As I mentioned in my letter in the last issue, ACM has decided to migrate Queue to the Web. As of July 2008, Queue will expand its publication frequency to 10 issues per year and publish those issues online using the most cutting-edge digital-editions technology available, as well as revamp the existing Queue Web site to provide an overall improved user experience.

Queue's new Web site will expand beyond the magazine's core content to offer additional features and new sources of quality content, while also enabling and encouraging communication among respected members of the software development community. Queue's digital edition will look just like the print edition you have been receiving and will offer a number of useful features for enhanced navigation, search, linking, and browsing. You can view Queue in its new digital format by visiting http://mags.acm.org/queue/current.

Is there a way to receive ACM Queue articles in print?

Yes, as of July 2008 a selection of Queue articles will appear in the new Practice section of the Communications of the ACM, the flagship publication of the ACM, with a current circulation of more than 87,000. In an effort to make the Communications of the ACM more appealing to a broader readership, including software developers and other practitioners in the software industry, the magazine is undergoing a complete redesign and editorial restructuring. These changes are also scheduled to debut in July.

If you are not already an ACM Member or Communications subscriber, there are two easy ways to receive it:

1. Join. ACM Professional Membership includes a complimentary subscription to Communications of the ACM magazine. (Note students can also receive a print version of Communications by selecting the appropriate student membership type.) ACM Professional Membership offers a host of additional career-enhancing benefits including: - Unlimited access to 2,500 online courses from SkillSoft

- Unlimited access to 1,100 online books from Safari (featuring a large selection from O'Reilly) and Books24x7

A bigger,
BETTER ONLINE
PRESENCE
FOR QUEUE

- Discounts on ACM SIG conference registration

- Full access to ACM's new Career \& Job Center with hundreds of targeted job postings

- TechNews and CareerNews e-mail digests and MemberNet newsletter

- The ACM Guide with more than 1 million bibliographic citations

- A free acm.org e-mail forwarding address with highquality Postini spam filtering

Learn more and join at: http://www.acm.org/ joinacm 2 .

2. Subscribe. Communications of the ACM is now available at a lower rate for individual subscribers: http://www.acm. org/addpubs.

Once again, thank you for making ACM Queue one of the most highly regarded resources in the computing industry. You've helped us shape a unique magazine, and we look forward to your continued input as we move forward with the Queue digital edition and the new Queue Web site, which is also scheduled to launch in July 2008.

Have questions or feedback? We'd love to hear from you via e-mail atqueue@acm.org. 